\title{
Natural products in drug discovery and human health
}

\author{
Maria-José U. Ferreira
}
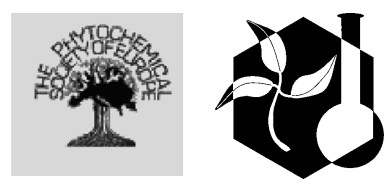

Published online: 2 January 2021

(C) The Author(s), under exclusive licence to Springer Nature B.V. part of Springer Nature 2021, corrected publication 2021

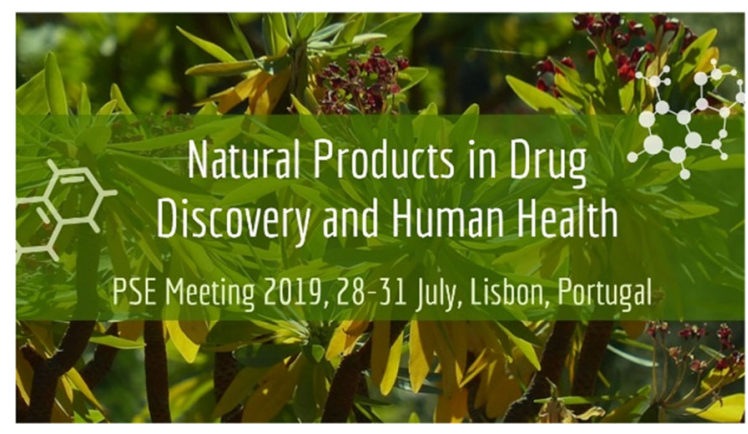

Natural Products and derived compounds play an important role in drug discovery by providing lead scaffolds, which can be optimized, by synthetic and medicinal chemists, in order to find effective agents to treat various diseases.

Aiming at discussing this subject, the Phytochemical Society of Europe (PSE) organized the conference of Natural Products in Drug Discovery and Human Health (NatProdDDH), which was held in Lisbon, Portugal, on July 28-31, 2019.

During 4 days, the conference, hosted by Faculty of Pharmacy, University of Lisbon, congregated the

M.-J. U. Ferreira ( $\square)$

Research Institute for Medicines (iMed.ULisboa), Faculty of Pharmacy, Universidade de Lisboa, Av. Prof. Gama Pinto, 1649-003 Lisbon, Portugal

e-mail: mjuferreira@ff.ulisboa.pt exciting world of Natural Products research, bringing together senior scientists, young researchers and $\mathrm{PhD}$ students, from Academy and Industry, from all over the world to share their latest advances.

The program of NatProdDDH covered the state-ofthe-art in Natural Products research, up to their application as value-added products for human health. Through plenary sessions, lectures, poster presentations, the conference provided the opportunity for the generation of innovative ideas, networking, and synergies.

It gathered 270 attendees from 37 countries (Europe, Asia, Africa and North and South America) and was organized in 17 sessions, that included 19 plenary lectures and 8 invited lectures by leading scientists, 53 oral communications, and two sessions where 165 posters were presented. To recognize the young scientific talents, five prizes were awarded to the best oral and poster presentations. Furthermore, five PSE travel bursaries were attributed to young researchers to attend the conference.

This special issue, constituted by 13 reviews based on some lectures of plenary and selected oral speakers of the conference, aims at illustrating some topics of the conference that dealt with several relevant features of ongoing natural products research, including applied aspects in human health.

Multidrug resistance (MDR) remains a major challenge in cancer chemotherapy. One of the main 
MDR mechanisms involves ATP-binding cassette $(\mathrm{ABC})$ transporter proteins, such as P-glycoprotein (P-gp/ABCB1), breast cancer resistance protein (BCRP/ABCG2), and ABCB5, which act as efflux pumps for anticancer drugs, decreasing their intracellular concentration. Several strategies for overcoming ABC-transporters-mediated MDR have been addressed, namely the development of ABC transporter inhibitors, mostly P-gp inhibitors, and collateral sensitivity agents characterized by their ability to selectively kill resistant cancer cells. Efferth et al. (2020) reviewed their work, developed during the last 20 years on different medicinal plants, aiming at finding bioactive natural product-derived compounds for tackling multidrug resistant cells.

Antibiotic resistance is one of the major threats to human health. New mechanisms of resistance are emerging and spreading worldwide. Currently, many of the available antibiotics are no longer effective, threatening the treatment of common infectious diseases and making bacterial infections the main cause of morbidity and mortality globally. Many bacterial species, such as Campylobacter jejuni, the most prevalent foodborne pathogen, can develop biofilms as a survival strategy in hostile environments. Klančnik et al. (2020) reviewed the anti-adhesion activities of phytochemicals against $C$. jejuni, the methodologies to evaluate the anti-adhesion effects, the mechanisms of adhesion, the first step in biofilm formation, and targets for reduction and control of $C$. jejuni in food processing environments.

Marine natural products from microbial origins have been considered promising sources of lead compounds in drug discovery. Among marine natural products, metabolites isolated from marine fungi, bearing high chemical diversity, have shown relevant biological activities, namely antimicrobial activity. Wang et al. (2020) reviewed the compounds isolated from marine fungi, reported in the last 20 years, which include polyketides, nitrogen-containing compounds, sterols, terpenoids and halogen-containing compounds, being the former compounds the main class of marine natural products. Their antimicrobial activities are also highlighted.

Diabetes is a metabolic disorder characterized by a high blood sugar level during a prolonged period of time. This disease occurs when the human body does not produce sufficient insulin, responsible for maintaining normal glucose index, or when the body cannot effectively use it. Protein tyrosine phosphatase 1B (PTP1B) is an emerging therapeutic target for the treatment of both type- 2 diabetes and obesity. PTP1B is involved in the down-regulation of insulin and leptin signaling. Due to its therapeutic potential, PTP1B has attracted a great interest and several selective and potent PTP1B inhibitors have been developed. Nazir et al. (2020) review focused on describing PTP1B inhibitors of secondary metabolites isolated from fungi, and their potential as anti-diabetic agents.

The $\alpha$-glucosidase enzyme catalyzes the final step in the digestion of carbohydrates by cleaving disaccharides and oligosaccharides into monosaccharides. In the control of type- 2 diabetes, one of the main strategies for decreasing postprandial hyperglycemia is to inhibit $\alpha$-glucosidase, thus delaying the absorption of glucose and, consequently, decreasing the necessity of insulin. Therefore, there is a great interest in developing $\alpha$-glucosidase inhibitors, which, currently, are considered as first-line drugs for treating type-2 diabetes. Hussain et al. (2020) reviewed $\alpha$ glucosidase inhibitors, reported in the last 40 years, isolated from fungi.

Type-2 diabetes, correlated with lifestyle and dietary patterns, and accounting for approximately $90 \%$ of diabetes worldwide, results from a lowered sensitivity of the insulin receptors. While many different dietary phytochemicals are believed to play a role in the glycaemic control and to prevent diabetesrelated complications, there mechanisms of action are not consensual. Three main mechanisms have been considered, namely their ability to reduce sugar uptake, to restore insulin function, or to attenuate the effects of oxidative stress and chronic inflammation. The review of Jin and Arroo (2020) address the role of dietary compounds in the prevention of type- 2 diabetes-related complications.

The review of Sánchez-Hidalgo et al. (2020) represents an overview of the literature about D-pinitol biosynthesis, plant distribution, biological activities and pharmacological studies, in vitro and in vivo, and clinical trials. D-Pinitol is a cyclitol found mainly in plants of the Leguminosae and Pinaceae families. Recently, there has been an increasing interest in cyclitols due to their presence in foods and medicinal plants. Particularly, several species containing D-pinitol have been traditionally used in the treatment of diabetes, inflammation, cancer and infection diseases. 
In case of diabetes, D-pinitol has been reported to have insulin-like properties, relieving insulin resistance.

Grauso et al. (2020) provided a critical review of the botanic characteristics, the traditional uses, the chemistry and pharmacology of Papaver rhoeas L. (Papaveraceae), contributing to the knowledge of this widespread plant, commonly known as corn poppy. It is an annual flowering medicinal plant that has been used since ancient times as food ingredient and in traditional medicine to treat nervousness, insomnia, digestive and respiratory disorders, baldness, eye infections and measles treatment. The chemical constituents of corn poppy have been largely studied. As a species of Papaveraceae family, alkaloids are the most representative secondary metabolites found in this weed plant, playing an important role on its biologic activity.

Aiming at valorizing Portuguese plants as source of bioactive compounds, Pinto and Silva (2020) reviewed their contribution to establish the chemical profile and to validate some of the health benefits claimed for some species, namely Calendula species, Pterospartum tridentatum (L.) Willk. and several halophytes growing in Portugal. They also focused on economically important species, which are at risk of being damaged by climate changes, such as Olea europaea L. and Eucalyptus globulus Labill., aiming at evaluating the impact that climate-change-related conditions can have in their metabolome profile.

Plants of Myrtaceae family have been used for a long time in traditional medicine and as food or spices. They are a source of bioactive compounds, namely phloroglucinols, a major class of natural compounds occurring in plants, microorganisms and marine organisms. Phloroglucinols are characterized by a wide structural variability that, owing to their bioactivity and structural features, have become attractive targets for organic chemists and pharmacologists. The review of Celaj et al. (2020) focuses on the chemical features of acylphloroglucinols and meroterpenoidacylphloroglucinols along with their biological activities. Additionally, synthetic procedures of myrtucommulones and derivatives are also addressed.

Plants of the Amaryllidaceae family have long been used in traditional medicine to treat various diseases. Their medicinal value has been attributed to the presence of Amaryllidaceae alkaloids, a group of plant metabolites with a wide range of biological properties, which are exclusively produced by this family. Among
Amaryllidaceae alkaloids, the haemanthamine-type alkaloids, represented by haemanthamine and haemanthidine, have received considerable attention, owing to their promising anticancer potential. Cahlíková et al. (2020) reviewed the reported data related to both alkaloids, including the preparation of semisynthetic derivatives.

D'yakonov et al. (2020) reviewed the current available data on the group of natural compounds containing bis-methylene-interrupted $Z$-double bonds in their scaffolds, including their biological activities and contents in different plants, animals, and microorganisms. This unique structural feature makes these compounds promising leads for developing antitumor, antibacterial, antimicrobial, and neuroprotective agents. The most efficient approaches for stereoselective formation of bis-methylene-interrupted $Z$ double bonds, and methods for the total synthesis of insect pheromones, acids, acetogenins, and alkynols, containing these fragments in the molecules, are discussed.

The indole alkaloid vincamanine, obtained from the leaves of Vinca minor L, is of particular interest for the treatment of neurodegenerative diseases, such as Parkinson and Alzheimer, and for producing the known vasodilating agent vinpocetine. Bonandi et al. (2020) reviewed the principal strategies for the total synthesis of vincamine, and related compounds, such as eburnamine, eburnamonine and apovincamine, sharing a fused pentacyclic scaffold. Moreover, the authors describe their own work related to the semisynthesis of $(+)$-vincamine, using tabersonine, a natural alkaloid extracted from Voacanga africana seeds, as starting material.

Acknowledgements We would like to thank all people who helped the local organizing committee to successfully accomplish the meeting, including the sponsors. We are also grateful to the Scientific Committee for their collaboration. We thank Faculty of Pharmacy, University of Lisbon, for hosting the conference, and the Portuguese Society of Chemistry for the financial management. Concerning this special issue, we greatly appreciated the work of the reviewers for their constructive criticisms for improving the papers. To conclude, we kindly acknowledge the editorial board of Phytochemistry Reviews, especially Prof. Rob Verpoorte for his invitation and support. 


\section{References}

Bonandi E, Foschi F, Marucci C et al (2020) Vincamine by synthesis and semi-synthesis. Phytochem Rev. https://doi. org/10.1007/s11101-020-09702-8

Cahlíková L, Kawano I, Řezáčová M et al (2020) The Amaryllidaceae alkaloids haemanthamine, haemanthidine and their semisynthetic derivatives as potential drugs. Phytochem Rev. https://doi.org/10.1007/s11101-02009675-8

Celaj O, Durán AG, Cennamo P, et al (2020) Phloroglucinols from Myrtaceae: attractive targets for structural characterization, biological properties and synthetic procedures. Phytochem Rev 8. https://doi.org/10.1007/s11101-02009697-2

D'yakonov VA, Dzhemileva LU, Dzhemilev UM (2020) Natural compounds with bis-methylene-interrupted Z-double bonds: plant sources, strategies of total synthesis, biological activity, and perspectives. Phytochem Rev. https://doi. org/10.1007/s11101-020-09685-6

Efferth T, Kadioglu O, Saeed MEM, et al (2020) Medicinal plants and phytochemicals against multidrug-resistant tumor cells expressing ABCB1, ABCG2, or ABCB5: a synopsis of 2 decades. Phytochemistry Rev. https://doi. org/10.1007/s11101-020-09703-7

Grauso L, de Falco B, Motti R, Lanzotti V (2020) Corn poppy, Papaver rhoeas L: a critical review of its botany, phytochemistry and pharmacology. Phytochem Rev. https://doi. org/10.1007/s11101-020-09676-7
Hussain H, Nazir M, Saleemet M, Al-Harrasi A et al (2020) Fruitful decade of Fungal Metabolites as Anti-diabetic agents from 2010-2019: Emphasis on $\alpha$-Glucosidase Inhibitors. Phytochem Rev

Jin Y, Arroo RRJ (2020) Application of dietary supplements in the prevention of type 2 diabetes-related cardiovascular complications. Phytochem Rev. https://doi.org/10.1007/ s11101-020-09710-8

Klančnik A, Šimunović K, Sterniša M et al (2020) Anti-adhesion activity of phytochemicals to prevent Campylobacter jejuni biofilm formation on abiotic surfaces. Phytochem Rev. https://doi.org/10.1007/s11101-020-09669-6

Nazir M, Saleem M, Ali I, Abbas G et al (2020) Fungal metabolites as Anti-diabetic agents: Emphasis on PTP1B inhibitors. Phytochem Rev

Pinto DCGA, Silva AMS (2020) Valorisation of Portuguese natural resources. Phytochem Rev. https://doi.org/10.1007/ s11101-020-09666-9

Sánchez-Hidalgo M, León-González AJ, Gálvez-Peralta M et al (2020) d-Pinitol: a cyclitol with versatile biological and pharmacological activities. Phytochem Rev. https://doi. org/10.1007/s11101-020-09677-6

Wang C, Tang S, Cao S (2020) Antimicrobial compounds from marine fungi. Phytochemostry Rev. https://doi.org/10. 1007/s11101-020-09705-5

Publisher's Note Springer Nature remains neutral with regard to jurisdictional claims in published maps and institutional affiliations. 